\title{
Nanocrystalline silicon dot displacement using speed-controlled tapping-mode atomic force microscopy
}

\author{
S. Kanjanachuchai ${ }^{\mathrm{a}, *}$, Y. Tsuchiya ${ }^{\mathrm{b}}$, K. Usami ${ }^{\mathrm{b}}$, S. Oda ${ }^{\mathrm{b}}$ \\ ${ }^{a}$ Semiconductor Device Research Laboratory, Department of Electrical Engineering, \\ Chulalongkorn University, Bangkok 10330, Thailand \\ ${ }^{\mathrm{b}}$ Research Center for Quantum Effect Electronics, Tokyo Institute of Technology, O-Okayama, Meguro-ku, Tokyo 152, Japan
}

Available online 18 March 2004

\begin{abstract}
Silicon nanocrystals in the scale of $10 \mathrm{~nm}$ have been grown using very-high-frequency chemical vapour deposition technique. While dot sizes could be well controlled, their distribution on silicon surface was found to be random. The as-grown nanocrystal $\mathrm{Si}$ dots do not adhere well to the substrate. It was found that non-contact probing of the surface using tapping-mode atomic force microscopy resulted in dot displacement. In speed-controlled experiments, dots can be relocated as far as $200 \mathrm{~nm}$ from their original locations. Furthermore, small surface structures can also be displaced. (C) 2004 Elsevier B.V. All rights reserved.
\end{abstract}

Keywords: Si nanocrystals; Speed-controlled tapping-mode AFM; Dot displacement

\section{Introduction}

The demonstration of silicon nanocrystals as a charge-storage element by Tiwari [1] has raised interests in several silicon-based nanostructure materials. Spherical nanocrystalline silicon (nc-Si) dots grown by very-high-frequency chemical vapour deposition have been shown to be capable of trapping electrons and, thus, are suitable for charge-storage purposes [2]. Despite good geometry control of the dots grown by such technique,

\footnotetext{
${ }^{*}$ Corresponding author.

E-mail address: songphol.k@chula.ac.th (S. Kanjanachuchai).
}

dot distribution on the substrate is still random. Attempts to line up the nc-Si dots using contactmode atomic force microscopy (AFM) have been reported [3]. In addition, contact-mode AFM has been used to inject charges into nc-Si dots [4] or to manipulate other types of nanostructures such as carbon nanotubes [5]. Decossas et al. have shown that it is possible to use an AFM tip for localized deposition, or for defining one-dimensional lines of nc-Si [6]. In this work, we were able to move ncSi dots using a non-contact, usually known as a tapping, mode AFM [7]. We show that, by controlling the speed of the scanning tip, tappingmode AFM can dislodge nanocrystals as large as $10 \mathrm{~nm}$ and surface structures as small as a few nanometers from their original positions. 


\section{Growth of nc-Si by VHFCVD}

Nanocrystalline silicon was deposited on $\mathrm{Si}$ substrates by very-high-frequency chemical vapour deposition (VHFCVD) pulsed plasma technique [3]. The system used in the growth consists of three main chambers: exchange chamber, plasma chamber and deposition chamber, as shown in Fig. 1. The plasma chamber has two inlets which supply $\mathrm{SiH}_{4}$ and $\mathrm{Ar}$ into the chamber. A small cylindrical outlet, $3 \mathrm{~mm}$ in diameter and $6 \mathrm{~mm}$ in length, connects the plasma chamber to the deposition chamber through a shutter.

The deposition follows the following steps. After the plasma chamber is pumped down to the base pressure of $10^{-10}$ Torr, $\mathrm{SiH}_{4}$ is supplied $(2 \mathrm{sccm})$. This raises the chamber pressure to $10^{-3}$ Torr, after which very high frequency (144 MHz) $\mathrm{RF}$ power of $3 \mathrm{~W}$ is supplied. This excites $\mathrm{SiH}_{4}$ and creates a plasma. When the plasma is stable, $\mathrm{Ar}$ is supplied in a cyclical on/off fashion: the valve which controls the Ar inlet turns on for $0.1 \mathrm{~s}$ and off for $1 \mathrm{~s}$, repeatedly. After many cycles, the nc-Si generation rate balances the rate at which nc-Si dots are pumped out of the chamber. The shutter is then opened and the nc-Si dots diffuse to the deposition chamber and land on the substrate. The amount of nc-Si deposited depends on the duration for which the shutter is open.

\section{Non-contact mode imaging results}

A Tapping-mode AFM image of as-grown nc-Si dots on a $\mathrm{Si}$ substrate is shown in Fig. 2(a). The image is taken in air with a $\mathrm{Si}_{3} \mathrm{~N}_{4}$ tip with a vibration frequency of approximately $140 \mathrm{kHz}$ using Seiko Instruments Inc's SPA400 with scanning speed of $1 \mathrm{~Hz}$ or $2.5 \mu \mathrm{m} / \mathrm{s}$. It can be seen from the figure that the spherical dots are distributed randomly throughout the substrate. The maximum dot height, and thus the diameter, in this area is approximately $10 \mathrm{~nm}$, and the dot density is $10^{9}$ $\operatorname{dots} / \mathrm{cm}^{2}$.

Another scan with varying scanning speeds, between 1 and $4 \mathrm{~Hz}$ (which corresponds to the tip

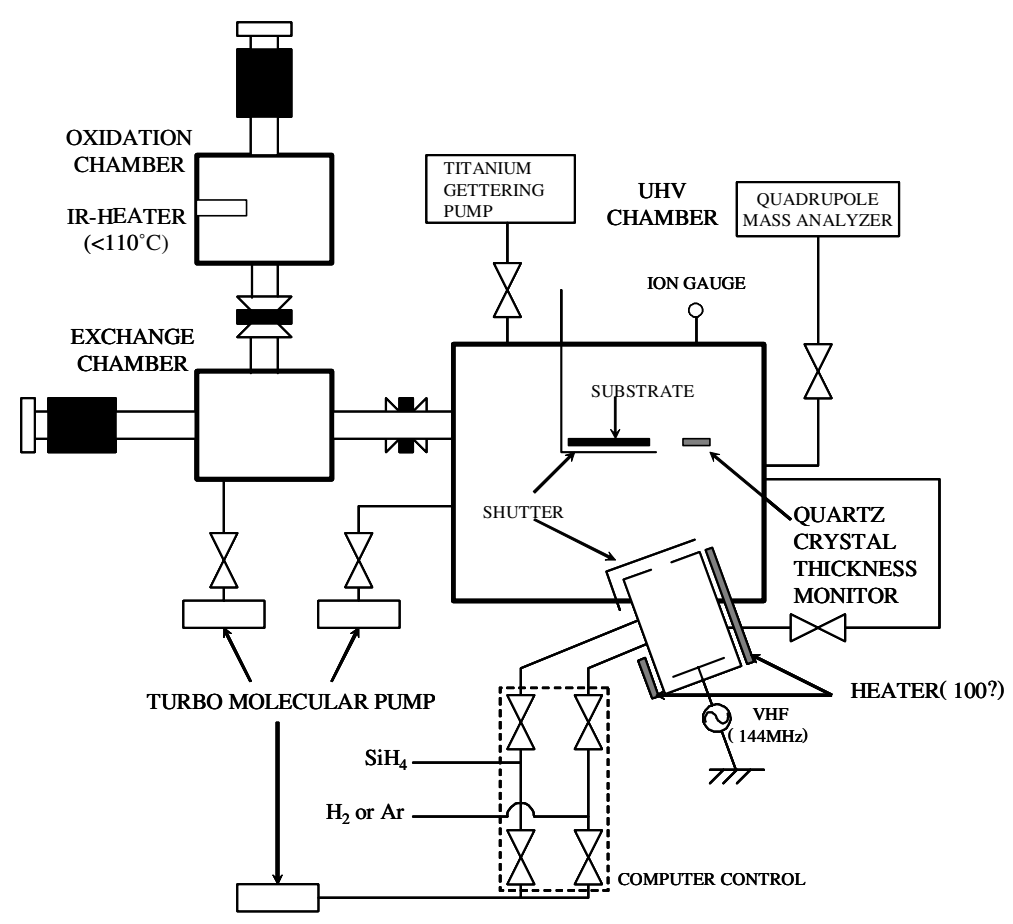

Fig. 1. Schematic drawing of the nc-Si deposition system. 

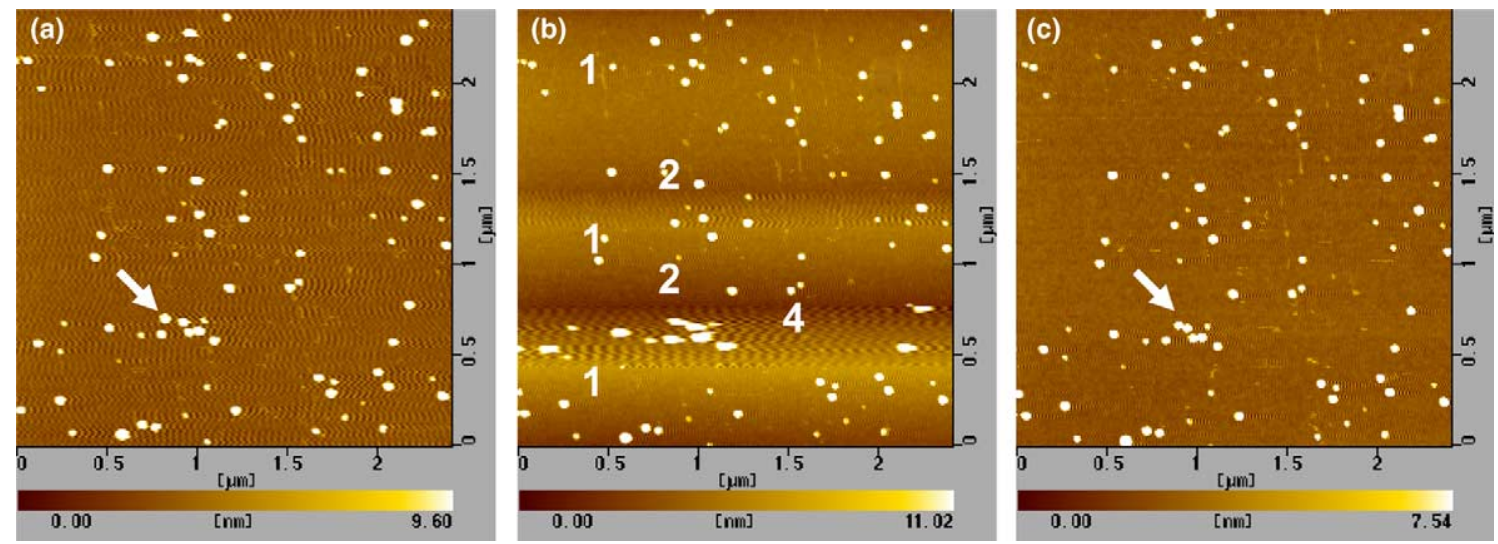

Fig. 2. Tapping-mode AFM images of as-grown nc-Si dots taken with scanning rate of: (a) $1 \mathrm{~Hz}$; (b) 1,2 and $4 \mathrm{~Hz}$ as indicated; (c) 1 Hz. The scanning rates of 1, 2 and $4 \mathrm{~Hz}$ correspond to tip speeds of 2.5, 5.0 and $10 \mu \mathrm{m} / \mathrm{s}$, respectively. (c) was taken right after (b). The arrows in (a) and (c) indicate the dot which was dislodged from its original position by the scanning procedure in (b). All fields are 2.5 $\mu \mathrm{m}$ square.

speed of $2.5-10 \mu \mathrm{m} / \mathrm{s}$ ), was then taken in the same area. The result is shown in Fig. 2(b). The blurring effect seen in the figure is a direct result of changing the scanning speed. Right after Fig. 2(b) was taken, another scan with a constant scanning speed of $1 \mathrm{~Hz}(2.5 \mu \mathrm{m} / \mathrm{s})$ was taken. The result of the final scan is shown in Fig. 2(c).

Close observation of the above figures shows that the final scan in Fig. 2(c) differs from the first scan in Fig. 2(a) in only one location, indicated by the arrows. It is clear that the indicated dot was moved by the scanning procedure performed when imaging Fig. 2(b). However, the areas which were scanned at 1 and $2 \mathrm{~Hz}$ were not affected regardless of the number of scans carried out at such speeds. It is therefore reasonable to conclude that scanning at a pace equal to or slower than $2 \mathrm{~Hz}(5 \mu \mathrm{m} / \mathrm{s})$ does not affect the locations of the nanostructures on the substrate. Only when scanning is performed at a faster pace do dots have the possibility to move. However, most dots in the areas which were scanned at $4 \mathrm{~Hz}(10 \mu \mathrm{m} / \mathrm{s})$ remain intact. This shows that only loose dots can be moved by the AFM tip. Similar experiments in different areas indicate that most dots stick to the substrate quite well. Only loose dots are dislodged from their original positions by fast sweeping of the AFM tip.

Certain surface structures with heights as small as a few nanometers can be dislodged also by non-contact mode AFM. Fig. 3(a) was taken at $2 \mathrm{~Hz}$, after which Fig. 3(b) was taken with scanning speeds of $2 \mathrm{~Hz}$ (top and bottom of the figure) or $8 \mathrm{~Hz}$ (middle band as indicated in the figure). Immediately afterwards, a $2-\mathrm{Hz}$ scan was taken, with the result shown in Fig. 3(c). The scanning rates of 2 and $8 \mathrm{~Hz}$ in these figures correspond to tip speeds of 0.6 and $4.8 \mu \mathrm{m} / \mathrm{s}$, respectively. While all dots remain intact, small surface structures in the middle and to the left of the image were displaced slightly. These structures clearly are not dots and may originate from contamination, which explains why they are not attached firmly to the underlying $\mathrm{Si}$ substrate. Since the tip vibrates at a height greater than 10 $\mathrm{nm}$ from the substrate and the height of the small surface structures is well below $10 \mathrm{~nm}$, we believe that the mechanisms that cause the displacement originate from either electrostatic repulsion, if the small surface structures are charged, or a force generated by difference in air pressures around the small structures caused by fast scanning, or a combination of both.

The faster the scanning speed, the easier and the further loose dots can be moved. Fig. 4(a) and (b) are images of the same area before and after fast scanning. The dotted rectangles in the figures are guide to the eye: they indicate the same location but have different sizes due to the 

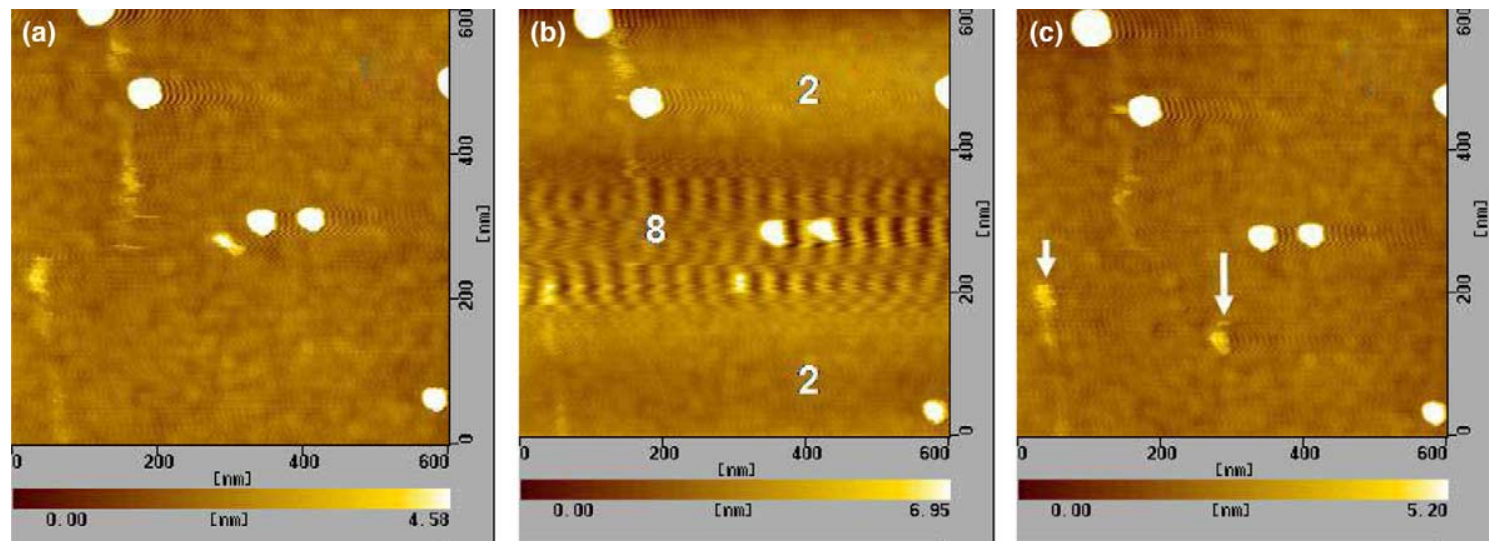

Fig. 3. Tapping-mode AFM images of as-grown nc-Si dots taken with scanning rate of: (a) $2 \mathrm{~Hz}$; (b) 2 and $8 \mathrm{~Hz}$ as indicated; (c) $2 \mathrm{~Hz}$. The scanning rates of 2 and $8 \mathrm{~Hz}$ correspond to tip speeds of 0.6 and $4.8 \mu \mathrm{m} / \mathrm{s}$, respectively. (c) was taken right after (b). The arrows indicate small surface structures which were dislodged from their original positions by the scanning procedure in (b). All fields are $600 \mathrm{~nm}$ square.
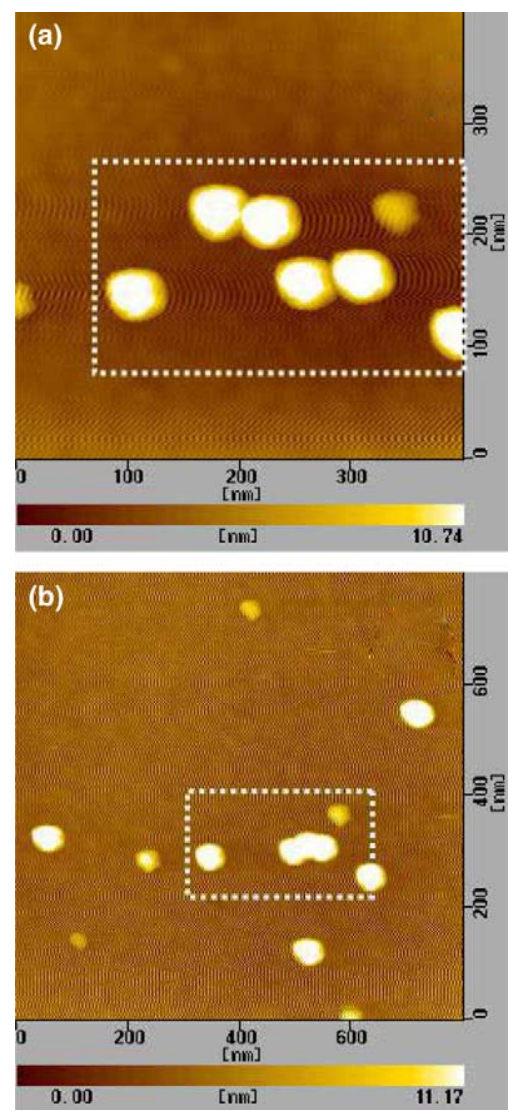

Fig. 4. Tapping-mode AFM images nc-Si dots in the same area taken with scanning rate of $2 \mathrm{~Hz}$ which corresponds to tip speeds of: (a) $0.8 \mu \mathrm{m} / \mathrm{s}$ (400 nm square field); (b) $1.6 \mu \mathrm{m} / \mathrm{s}(800$ nm square field). difference in scanned area. After Fig. 4(a) was taken at $2 \mathrm{~Hz}(0.8 \mu \mathrm{m} / \mathrm{s})$, the same area was scanned at $16 \mathrm{~Hz}(12.8 \mu \mathrm{m} / \mathrm{s}$, not shown). The final $2-\mathrm{Hz}$ scan to cover a larger area, hence with a corresponding higher tip speed of $1.6 \mu \mathrm{m} / \mathrm{s}$, was then carried out and results shown in Fig. 4(b). It can be seen in the figures that, within the viewing range of $400 \mathrm{~nm} \times 400 \mathrm{~nm}$, the four separate dots coalesce into a 3-dot ensemble and the other dot is missing from the current view. Further scanning to cover a much larger area around the boxed region shows that the missing dot is in fact relocated to approximately $200 \mathrm{~nm}$ south of its original position.

The above results show that atomic manipulation of as-grown nc-Si dots via tapping mode AFM is possible. However, the lifetime of the scanning tip is significantly reduced if it is used to dislodge loose structure on the substrate. It was found that some tips were damaged after a few scans at fast speed $(>10 \mu \mathrm{m} / \mathrm{s})$, particularly when scanning over large dots with diameters larger than $10 \mathrm{~nm}$. This is because, for the tapping mode, the height between the end of the scanning tip and the substrate is about $10 \mathrm{~nm}$. Fast scanning over a height greater than $10 \mathrm{~nm}$ means that the tip has not sufficient time to move up, and the tip end may come into contact or crash the underlying structure, resulting in movement of both the structure and the atoms 
at the apex of the tip. Once the tip loses its sharpness, the resolution of the scan image will be reduced.

One way to fix the nc-Si dots to the substrate is to subject the as-grown sample to thermal oxidation. However, the dot size will be reduced. This can be avoided by depositing an oxide passivation layer on top of the nc-Si.

\section{Conclusion}

In conclusion, we show that tapping-mode AFM can displace loose structures, large or small, from their original positions. Therefore, there exists a possibility of perturbing the surface being imaged by tapping-mode AFM, depending on scanning speed, the charge state of the nanostructures, air pressure, and the stickiness to the substrate of the structures being imaged.

\section{Acknowledgements}

This work was supported by CREST/JST, AFOSR and the Thai Ministry of University Affairs.

\section{References}

[1] S. Tiwari, F. Rana, H. Hanafi, A. Hartstein, E.F. Crabbé, K. Chan, Appl. Phys. Lett. 68 (1996) 1377.

[2] S.Y. Huang, S. Banerjee, R.T. Tung, S. Oda, J. Appl. Phys. 93 (1) (2003) 576.

[3] S. Oda, Adv. Colloid Interf. Sci. 71-72 (1997) 31.

[4] for example, see S. Banerjee, M.A. Salem, S. Oda, Appl. Phys. Lett. 83 (2003) 3788;

E.A. Boer, L.D. Bell, M.L. Brongersma, H.A. Atwater, M.L. Ostraat, R.C. Flagan, Appl. Phys. Lett. 78 (2001) 3133.

[5] J.Y. Park, Y. Yaish, M. Brink, S. Rosenblatt, P.L. McEuen, Appl. Phys. Lett. 80 (2002) 4446.

[6] S. Decossas, F. Mazen, T. Baron, G. Bremond, A. Souifi, Nanotechnology 14 (2003) 1272.

[7] R. Garcia, R. Perez, Surf. Sci. Rep. 47 (2002) 197. 